TITLE:

\title{
Application of stop and play models to the representation of magnetic characteristics of silicon steel sheet
}

\section{$\operatorname{AUTHOR}(\mathrm{S})$ :}

Matsuo, T; Shimode, D; Terada, Y; Shimasaki, M

\section{CITATION:}

Matsuo, T ... [et al]. Application of stop and play models to the representation of magnetic characteristics of silicon steel sheet. IEEE TRANSACTIONS ON MAGNETICS 2003, 39(3): 1361-1364

\section{ISSUE DATE:}

2003-05

URL:

http://hdl.handle.net/2433/39997

\section{RIGHT:}

(c)2003 IEEE. Personal use of this material is permitted. However, permission to reprint/republish this material for advertising or promotional purposes or for creating new collective works for resale or redistribution to servers or lists, or to reuse any copyrighted component of this work in other works must be obtained from the IEEE. 


\title{
Application of Stop and Play Models to the Representation of Magnetic Characteristics of Silicon Steel Sheet
}

\author{
Tetsuji Matsuo, Member, IEEE, Daisuke Shimode, Yasushi Terada, and Masaaki Shimasaki, Member, IEEE
}

\begin{abstract}
The stop and play models are applied to the representation of the hysteretic characteristics of a silicon steel sheet. Both models are used to provide hysteretic functions from $B$ to $H$. The play model identified from symmetric $B-H$ loops describes the hysteretic characteristics much more accurately than does the stop model. Combining the two models achieves a more accurate representation than either of the two models alone.
\end{abstract}

Index Terms-Hysteresis, identification, play and stop models, silicon steel sheet.

\section{INTRODUCTION}

INCE THE stop model [1]-[4] can provide a hysteretic $\checkmark$ output of magnetic field $H$ from an input of magnetic flux density $B$, it can be an efficient tool for magnetic-field analyses using magnetic vector potential. A previous study [4] has proposed an identification method of the stop model from measured $B-H$ loops and has shown that the stop model can successfully simulate an inverse of the Preisach model.

The ability of the stop model to describe magnetic characteristics of ferromagnetic materials, however, has not been sufficiently examined since the identification method in [4] has not yet been applied to ferromagnetic materials.

The play model [1], [2], on the other hand, has been proven to be able to represent hysteretic characteristics equivalently to the scalar static Preisach model [5], [6]. The play model and the Preisach model usually give $B$ from an input $H$. The inverse distribution function method proposed in [7], however, enables the Preisach model to provide an output $H$ from $B$. In the same way, the play model can also provide $H$ from $B$ because of the equivalence [2] to the Preisach model.

This paper first compares the ability of the stop and play models to represent hysteretic relations from $B$ to $H$ in a silicon steel sheet. The present paper then proposes a combination of the stop and play models to improve the hysteretic representation.

Manuscript received June 18, 2002. This work was supported in part by the Kawasaki Steel 21st Century Foundation.

T. Matsuo, Y.Terada, and M. Shimasaki are with the Graduate School of Engineering, Kyoto University, Kyoto, 606-8501, Japan (e-mail: tmatsuo@kuee.kyoto-u.ac.jp; simasaki@kuee.kyoto-u.ac.jp)

D. Shimode was with the Graduate School of Engineering, Kyoto University, Kyoto 606-8501, Japan. He is now with Central Japan Railway Company, Nagoya 450-6101, Japan.

Digital Object Identifier 10.1109/TMAG.2003.81017

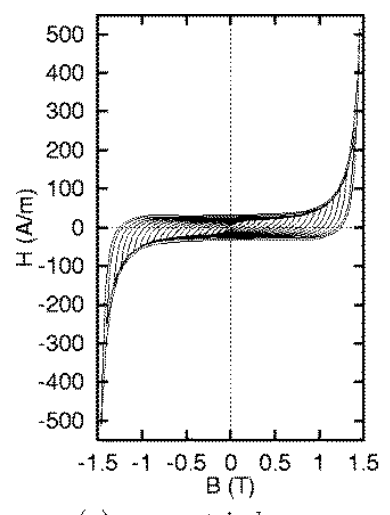

(a) symmetric loops

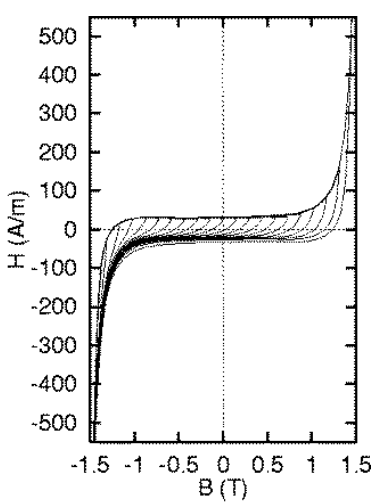

(b) asymmetric loops
Fig. 1. Measured magnetic characteristics of 50A290.

\section{Application OF Stop Model}

The scalar stop model [1]-[4] describes a hysteretic relation between $B$ and $H$ as

$$
H(B)=\sum_{k=1}^{M} g_{k}\left(s_{k}(B)\right)
$$

where $s_{k}$ is the stop hysteron operator, $M$ is the number of hysteron operators, and $g_{k}$ is the shape function. The stop hysteron operator $s_{k}$ is given by

$$
s_{k}(B)=\max \left(\min \left(B-B^{0}+s_{k}^{0}, \eta_{k}\right),-\eta_{k}\right)
$$

where $B^{0}$ and $s_{k}^{0}$ are the values of $B$ and $s_{k}$ at the previous time-point, and $\eta_{k}$ is a constant.

This paper sets $\eta_{k}$ to $k B_{s} / M(k=1, \ldots, M)$ where $B_{s}$ is a saturation magnetic flux density. The shape function $g_{k}$ is assumed to be piecewise linear as

$g_{k}(s)=g_{k}\left(s_{k, j-1}\right)+\kappa_{k, j} \cdot\left(s-s_{k, j-1}\right)\left(s_{k, j-1} \leq s \leq s_{k, j}\right)$

where $s_{k, j}=-\eta_{k}+j \Delta s(j=0, \ldots, k), \Delta s=2 B_{s} / M$, and $\kappa_{k, j}=\left\{g_{k}\left(s_{k, j}\right)-g_{k}\left(s_{k, j-1}\right)\right\} / \Delta s$. The identification procedure proposed in [4] yields $\kappa_{k, j}$ from measured $B-H$ loops using the same number of stop hysterons as that of the measured loops (see Appendix I).

The magnetic characteristics of a nonoriented silicon steel sheet (JIS: 50A290) are measured by a single sheet tester [8], [9]. Fig. 1 shows two kinds of $B-H$ loops with amplitudes $k B_{s} / N\left(k=1,2, \ldots, N, N=20\right.$, and $\left.B_{s}=1.46 \mathrm{~T}\right)$ measured at $1 \mathrm{~Hz}$, one of which is symmetric loops [Fig. 1(a)], the other being $B-H$ loops that make first-order reversal curves from the 


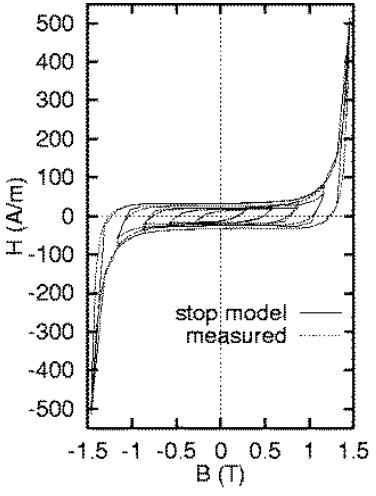

(a) symmetric loops

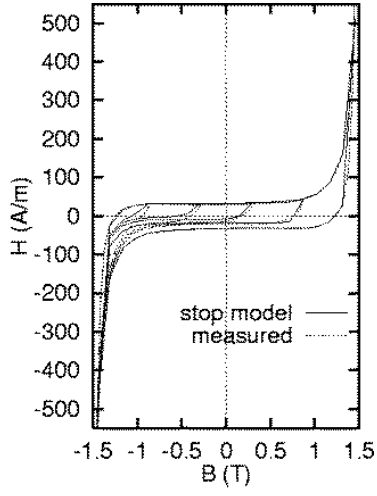

(b) asymmetric loops
Fig. 2. Stop model identified from symmetric loops.

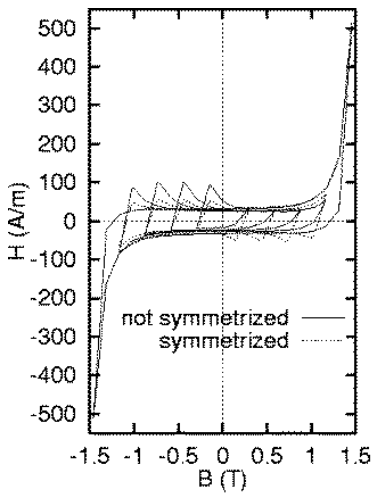

(a) symmetric loops

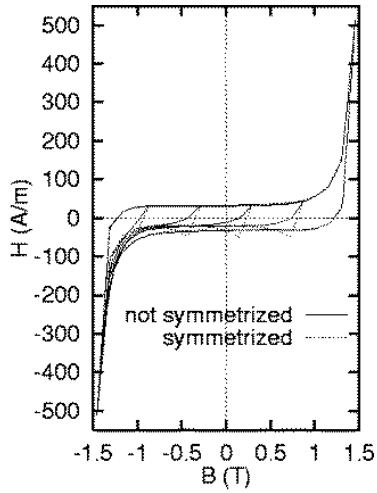

(b) asymmetric loops
Fig. 3. Stop model identified from asymmetric loops.

negative saturation [Fig. 1(b)]. The present paper calls the latter loops "asymmetric loops" for simplicity.

$B-H$ loops simulated by the stop model identified from the symmetric loops are shown in Fig. 2, where the identification from the symmetric loops results in inaccurate representation of the asymmetric loops.Fig. 2 also shows that sharp saturation of $B$ is not smoothly represented because the number of nodes of the piecewise linear function (3) is insufficient.

Fig. 3 shows $B-H$ loops given by the stop model identified from the asymmetric loops, where its symmetrized characteristics [4] are also shown.Fig. 3 shows that the identification from the asymmetric loops yields $B-H$ loops that differ greatly from the measured symmetric loops and that the symmetrization does not improve the representation.

This discrepancy between the measured and simulated loops is caused by the property of equal vertical chords [4] that is required for the hysteretic representation by the stop model. The comparison of Fig. 1(a) and (b) shows that the widths of the asymmetric loops along the $B$ direction are much larger than those of the symmetric loops, which means that this steel sheet does not show the property of equal vertical chords.

\section{ApPlication of the Play Model}

The play model [1], [2] is also able to provide a hysteretic output of $H$ from an input $B$ in the same way as does the Preisach model [5], [6] using the inverse distribution function method [7].

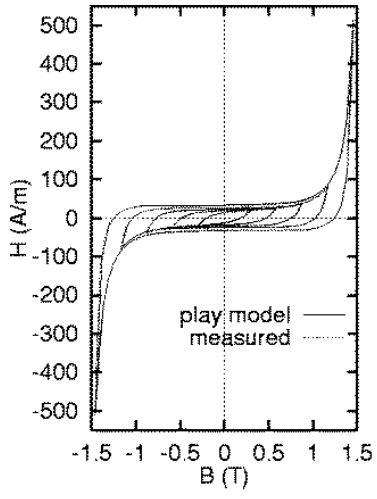

(a) symmetric loops

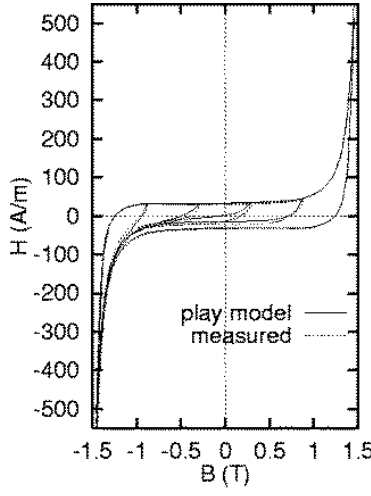

(b) asymmetric loops
Fig. 4. Play model identified from symmetric loops.

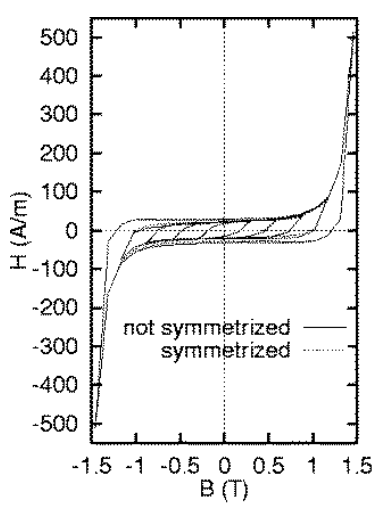

(a) symmetric loops

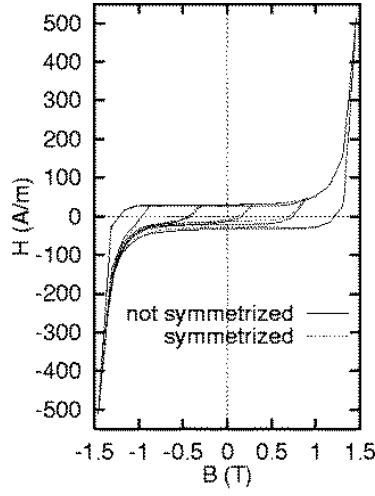

(b) asymmetric loops
Fig. 5. Play model identified from asymmetric loops.

The play model describes a hysteretic relation between $H$ and $B$ as

$$
H(B)=\sum_{k=1}^{M} f_{k}\left(p_{k}(B)\right)
$$

where $p_{k}$ is the play hysteron operator, $M$ is the number of hysteron operators, and $f_{k}$ is the shape function. The play hysteron operator $p_{k}$ is given by

$$
p_{k}(B)=\max \left(\min \left(p_{k}^{0}, B+\zeta_{k}\right), B-\zeta_{k}\right)
$$

where $p_{k}^{0}$ is the value of $p_{k}$ at the previous time-point and $\zeta_{k}$ is a constant.

Setting $\zeta_{k}=(k-1) B_{s} / M(k=1, \ldots, M)$, this paper assumes that the shape function $f_{k}$ is piecewise linear as

$f_{k}(p)=f_{k}\left(p_{k, j-1}\right)+\mu_{k, j} \cdot\left(p-p_{k, j-1}\right)\left(p_{k, j-1} \leq p \leq p_{k, j}\right)$

where $p_{k, j}=-B_{s}+\zeta_{k}+j \Delta p(j=0, \ldots, M-k+1)$, $\Delta p=2 B_{s} / M$, and $\mu_{k, j}=\left\{f_{k}\left(p_{k, j}\right)-f_{k}\left(p_{k, j-1}\right)\right\} / \Delta p$.

Because of the equivalence [2] to the Preisach model, the identification method for the Preisach model from the Everett function [6] can be applied to the play model. The shape functions of the play model are determined from symmetric $B-H$ loops (see Appendix II) in a similar way to the identification from asymmetric loops (i.e., first-order reversal curves from the negative saturation). It is noted that the identification of the play model from $N$ symmetric loops uses $2 N$ play hysterons 


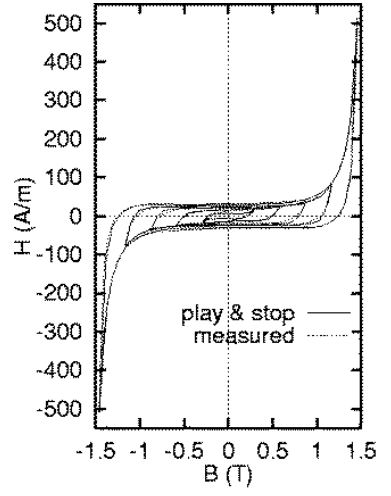

(a) symmetric loops

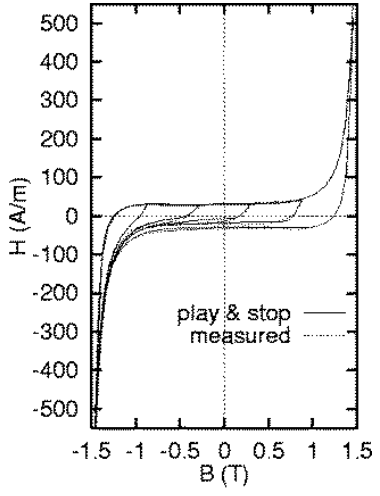

(b) asymmetric loops
Fig. 6. Combination model $C_{1}$.

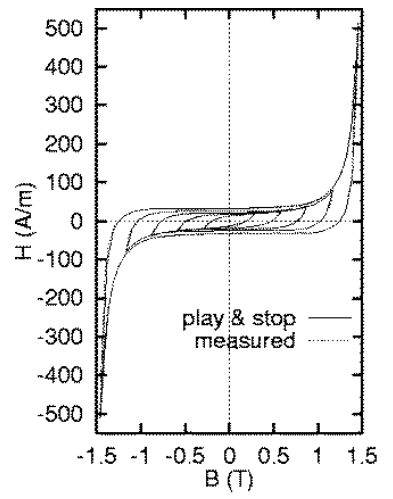

(a) symmetric loops

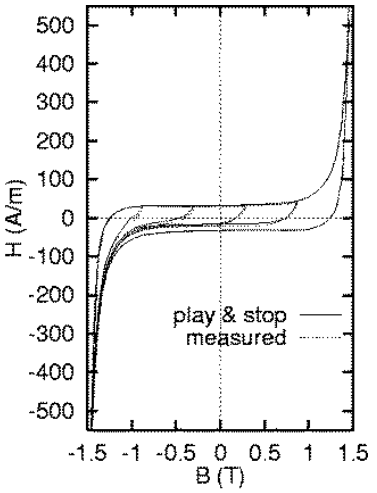

(b) asymmetric loops
Fig. 7. Combination model $C_{2}$.

whereas the identification from $N$ asymmetric loops requires $N$ play hysterons.

$B-H$ loops simulated by the play model identified from the symmetric loops are shown in Fig. 4, where the identification from the symmetric loops gives more accurate representation of the symmetric loops than the stop model because of the increase in the number of hysterons used. Small discrepancy is seen between the measured and simulated asymmetric loops in Fig. 4(b).

Fig. 5 shows $B-H$ loops given by the play model identified from the asymmetric loops, where its symmetrized characteristics are also shown. Fig. 5 shows that the sharp saturation of $B$ is not smoothly represented because of the insufficient number of hysterons. Fig. 5(a) shows that the identification from the asymmetric loops results in asymmetric representation of the symmetric loops and that the symmetrization improves the representation of the symmetric loops.

\section{Combination of Play and Stop Models}

The preceding sections have shown that neither the stop nor the play model gives a complete representation of both the symmetric and asymmetric loops. This section examines a combination of both models in order to improve the hysteretic representation.

Since the play model identified from the symmetric loops seems to most accurately represent the hysteretic characteristics of the silicon steel sheet in the preceding sections, the com-

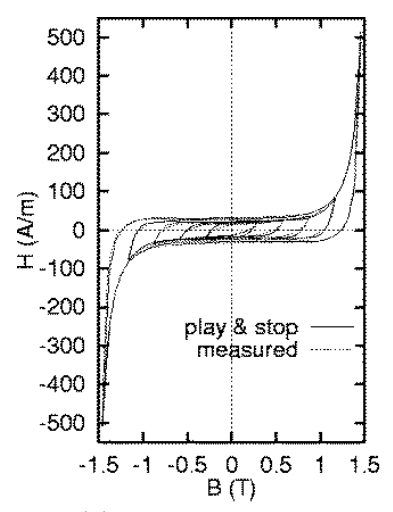

(a) symmetric loops

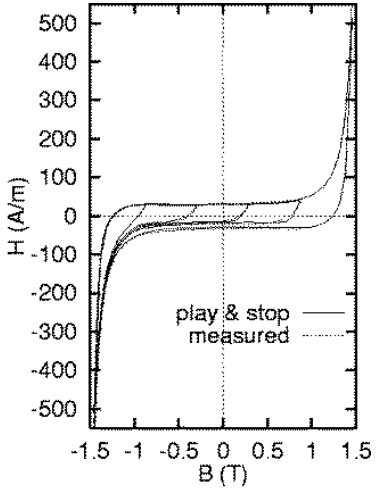

(b) asymmetric loops
Fig. 8. Combination model $C_{9}$.

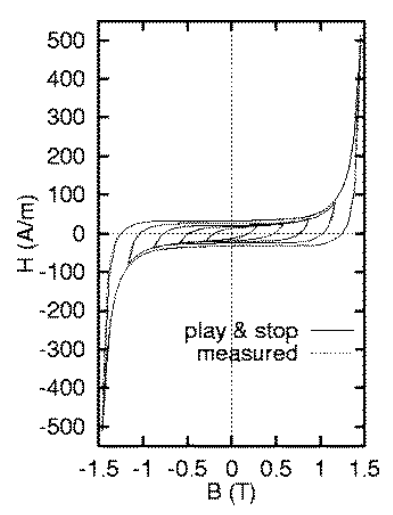

(a) symmetric loops

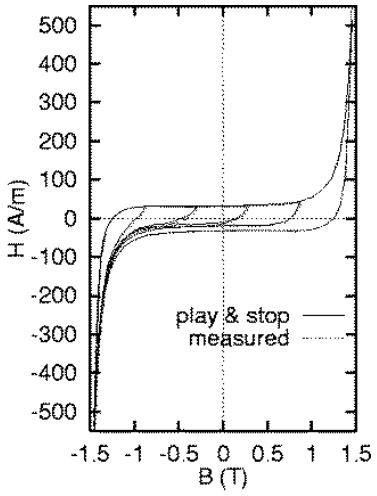

(b) asymmetric loops
Fig. 9. Combination model $C_{10}$.

bination model is based on the play model identified from the symmetric loops and it is combined with the stop model that is identified from the asymmetric loops and symmetrized.

Let $P(f(B))$ be the hysteretic function that is represented by the play model identified from the symmetric loops of a hysteretic function $f(B)$. Similarly, let $S(f(B))$ be the hysteretic function that is represented by the stop model identified from the asymmetric loops of $f(B)$ with the symmetrization.

The combination models are constructed from measured hysteretic characteristics $h(B)$ as follows. The first approximation of combination model $C_{0}$ is given by the play model

$$
C_{0}(B)=P(h(B)) .
$$

Next, the difference between $h(B)$ and $C_{0}(B)$ is represented by the stop model and added to $C_{0}(B)$

$$
C_{1}(B)=C_{0}(B)+S\left(h(B)-C_{0}(B)\right) .
$$

Combination models $C_{i}(B)$ are then sequentially generated as

$$
\begin{aligned}
C_{2 i}(B) & =C_{2 i-1}(B)+P\left(h(B)-C_{2 i-1}(B)\right) \\
C_{2 i+1}(B) & =C_{2 i}(B)+S\left(h(B)-C_{2 i}(B)\right) .
\end{aligned}
$$

Figs. 6-9 show the hysteretic characteristics given by the combination models $C_{1}, C_{2}, C_{9}$, and $C_{10}$, respectively.

Further generations of $C_{2 i-1}$ and $C_{2 i}(i>5)$ do not significantly improve the hysteretic representation compared with $C_{9}$ and $C_{10}$, respectively. Fig. 10 shows averaged characteristics 


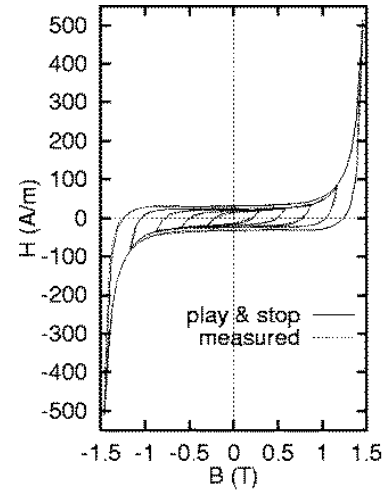

(a) symmetric loops

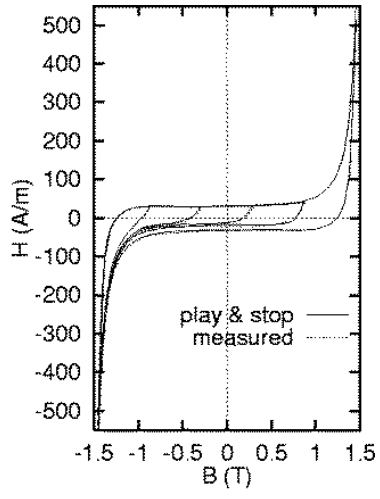

(b) asymmetric loops

Fig. 10. Combination model $\left(C_{9}+C_{10}\right) / 2$.

$\left(C_{9}(B)+C_{10}(B)\right) / 2$, which reconstruct the measured loops precisely.

\section{APPENDIX I}

IDENTIFICATION OF STOP MODEL

Equation (18) in [4] is incorrect. It should be

$$
R_{1}^{\prime}\left(\eta_{k}, s_{k, j}\right)= \begin{cases}0, & (k=j=0) \\ R_{1 m}^{\prime}\left(\eta_{k}, s_{k, j}\right), & (1 \leq k \leq M, 0 \leq j \leq k) \\ R_{1 M}^{\prime}\left(s_{M, j}\right), & (k=M+1,1 \leq j \leq M)\end{cases}
$$

$R_{1 m}^{\prime}\left(\eta_{k}, s_{k, j}\right)=h_{+}\left(\eta_{k}, s_{k, j}+x_{0 k}\right)-h_{-}\left(\eta_{k}, s_{k, j}+x_{0 k}\right)$

$$
R_{1 M}^{\prime}\left(s_{M, j}\right)=h_{+}\left(\eta_{M}, s_{M, j}\right)-h_{-}\left(\eta_{M}, s_{M, j-1}\right)
$$

where $x_{0 k}$ is the dc bias of periodic input (7) in [4] with the amplitude $\eta_{k}$ and $h_{+}(\eta, x)$ and $h_{-}(\eta, x)$ are the ascending and descending curves of hysteresis loop with the amplitude $\eta$, respectively. The function $R_{1}^{\prime}$ gives $\kappa_{k, j}$ as

$$
\begin{aligned}
\kappa_{k, j}=\frac{1}{\Delta s}\{ & R_{1}^{\prime}\left(\eta_{k+1}, s_{k+1, j}\right)-R_{1}^{\prime}\left(\eta_{k}, s_{k, j}\right) \\
& \left.-R_{1}^{\prime}\left(\eta_{k}, s_{k, j-1}\right)+R_{1}^{\prime}\left(\eta_{k-1}, s_{k-1, j-1}\right)\right\} .
\end{aligned}
$$

\section{APPENDIX II \\ IDENTIFICATION OF THE PLAY MODEL FROM SYMMETRIC LOOPS}

Let $H_{+}\left(B_{a}, B\right)$ and $H_{-}\left(B_{a}, B\right)$ be the ascending and descending curves of symmetric loop with the amplitude $B_{a}$, respectively. The Everett function [6] $E\left(B_{A}, B_{B}\right)$ is defined from symmetric loops as

$$
\begin{aligned}
& E\left(B_{A}, B_{B}\right) \\
& =\left\{\begin{array}{ll}
H_{-}\left(B_{B}, B_{B}\right)-H_{-}\left(B_{B}, B_{A}\right) & \left(B_{A} \geq-B_{B}\right) \\
H_{+}\left(\left|B_{A}\right|, B_{B}\right)-H_{+}\left(\left|B_{A}\right|, B_{A}\right) & \left(B_{A}<-B_{B}\right)
\end{array} .\right.
\end{aligned}
$$

The Everett function gives $\mu_{k, j}$ as

$$
\begin{aligned}
\mu_{k, j}= & E\left(B_{j-1}, B_{i}\right)-E\left(B_{j-1}, B_{i-1}\right) \\
& -E\left(B_{j}, B_{i}\right)+E\left(B_{j}, B_{i-1}\right) \\
i= & k+j-1
\end{aligned}
$$

where $B_{n}=2 n B_{s} / M-B_{s}(n=0, \ldots, M)$.

\section{REFERENCES}

[1] M. A. Krasnosel'skii and A. V. Pokrovskii, Systems With Hysteresis. Berlin, Germany: Springer-Verlag, 1989.

[2] G. Miano, C. Serpico, and C. Visone, "A new model of magnetic hysteresis, based on stop hysterons: An application to the magnetic field diffusion," IEEE Trans. Magn., vol. 32, pp. 1132-1135, May 1996.

[3] S. Bobbio, G. Miano, C. Serpico, and C. Visone, "Models of magnetic hysteresis based on play and stop hysterons," IEEE Trans. Magn., vol. 33, pp. 4417-4426, Nov. 1997.

[4] T. Matsuo and M. Shimasaki, "Isotropic vector hysteresis represented by superposition of stop hysteron models," IEEE Trans. Magn., vol. 37, pp. 3357-3361, Sept. 2001. p. 3995, Nov.

[5] I. D. Mayergoyz, Mathematical Models of Hysteresis. Berlin, Germany: Springer-Verlag, 1991.

[6] E. D. Torre, Magnetic Hysteresis. New York: IEEE Press, 1999.

[7] N. Takahashi, S. Miyabara, and K. Fujiwara, "Problems in practical finite element analysis using Preisach hysteresis model," IEEE Trans. Magn., vol. 35, pp. 1243-1246, May 1999.

[8] H. Nishimoto, S. Miyabara, M. Nakano, K. Fujiwara, and N. Takahashi, "Evaluation method of magnetic properties of electrical steel sheets by using the Preisach model," in Pap. Technical Meeting Inst. Elec. Eng. Japan, vol. MAG-97-178, 1997, pp. 41-46.

[9] H. Nishimoto, M. Nakano, K. Fujiwara, and N. Takahashi, "Effect of frequency on magnetic properties," in Pap. Technical Meeting Inst. Elec. Eng. Japan, vol. MAG-98-170, 1998, pp. 21-26. 